\title{
DISPLACEMENT OF THE RUPTURED ULNAR COLLATERAL LIGAMENT OF THE METACARPO-PHALANGEAL JOINT OF THE THUMB
}

\author{
A Clinical and Anatomical Study \\ Bertil Stener, Gothenburg, Sweden \\ From the Department of Surgery of the Extremities and the Hand Service, \\ Sahlgrenska Sjukhuset, University of Gothenburg
}

\begin{abstract}
Having observed several cases in which, after distal rupture, the ulnar collateral ligament of the metacarpo-phalangeal joint of the thumb had become folded over to point proximally, I set about making a systematic investigation of the pathological anatomy of rupture of this ligament. The investigation was carried out (in connection with operation) upon thirtynine patients with total rupture of the ligament. In the great majority of cases the ligament was found to have been displaced to a greater or lesser extent; the rupture was most often distal, and in most of these cases the ulnar expansion of the dorsal aponeurosis was interposed between the ruptured end of the ligament and the site of its attachment to the phalanx. These observations provide a strong argument in favour of operative treatment when this ligament is ruptured. The clinical investigation was supplemented by an anatomical study of fresh post-mortem specimens.
\end{abstract}

\section{MATERIAL}

The clinical material comprised thirty-nine consecutive cases of total rupture of the ulnar collateral ligament of the metacarpo-phalangeal joint of the thumb (all of them operated upon by the author) and one case in which radiographs provided indirect evidence that the ligament had been displaced (Case 3). Of the total of forty patients, thirty-four were operated upon within sixteen days of the accident (recent cases). The other six patients (older cases) were operated upon after seven weeks, four months, five months, nine months, eight years and ten years.

The anatomical investigation was made on forty-two fresh post-mortem specimens.

\section{ANATOMY}

Figure 1 shows the dorsal aspect (A) and ulnar aspects (B and C) of the metacarpophalangeal joint of the thumb*. On a level with the joint, transverse fibres of the dorsal aponeurosis $(a)$ form the ulnar and radial expansions of this structure. The adductor pollicis muscle $(b)$ is inserted partly through the ulnar sesamoid bone (not visible here) and partly through a powerful tendon direct into the phalanx $(c)$; in addition, some of its fibres fuse with the ulnar expansion of the dorsal aponeurosis. This part of the dorsal aponeurosis is thus in fact a part of the tendon system of adductor pollicis (Mondry 1940) and has therefore been referred to in the present work as the adductor aponeurosis $(d)$. If one severs the adductor aponeurosis at right angles to the line of its fibres (C), one exposes the ulnar collateral ligament proper $(e)$ and the ulnar accessory collateral ligament $(f)$. The tendon of extensor pollicis longus $(g)$ is anchored through the adductor aponeurosis to the ulnar sesamoid bone which, besides belonging to the tendon system of adductor pollicis, is also incorporated in the palmar and collateral ligamentary apparatus of the joint (Fig. 2) (Stener 1962). When the joint is about half way between full extension and full flexion the proximal edge of the adductor aponeurosis is more or less level with the point where the proper and accessory ulnar collateral

* So as to make it easier to compare one case with another, all the illustrations have been made to represent a right thumb, even when it was in fact the left thumb that was injured.

VOl. 44 B, NO. 4, NOVEMBER 1962 
ligaments are attached to the metacarpal head (Fig. 1, B and C). The adductor aponeurosis is separated from the underlying ligaments by loose connective tissue. When the joint is flexed the dorsal part of the adductor aponeurosis is shifted in a distal direction together with the tendon of extensor pollicis longus, while the palmar part is at the same time shifted proximally together with the sesamoid bone (Fig. 2); the opposite is the case when the joint is extended.
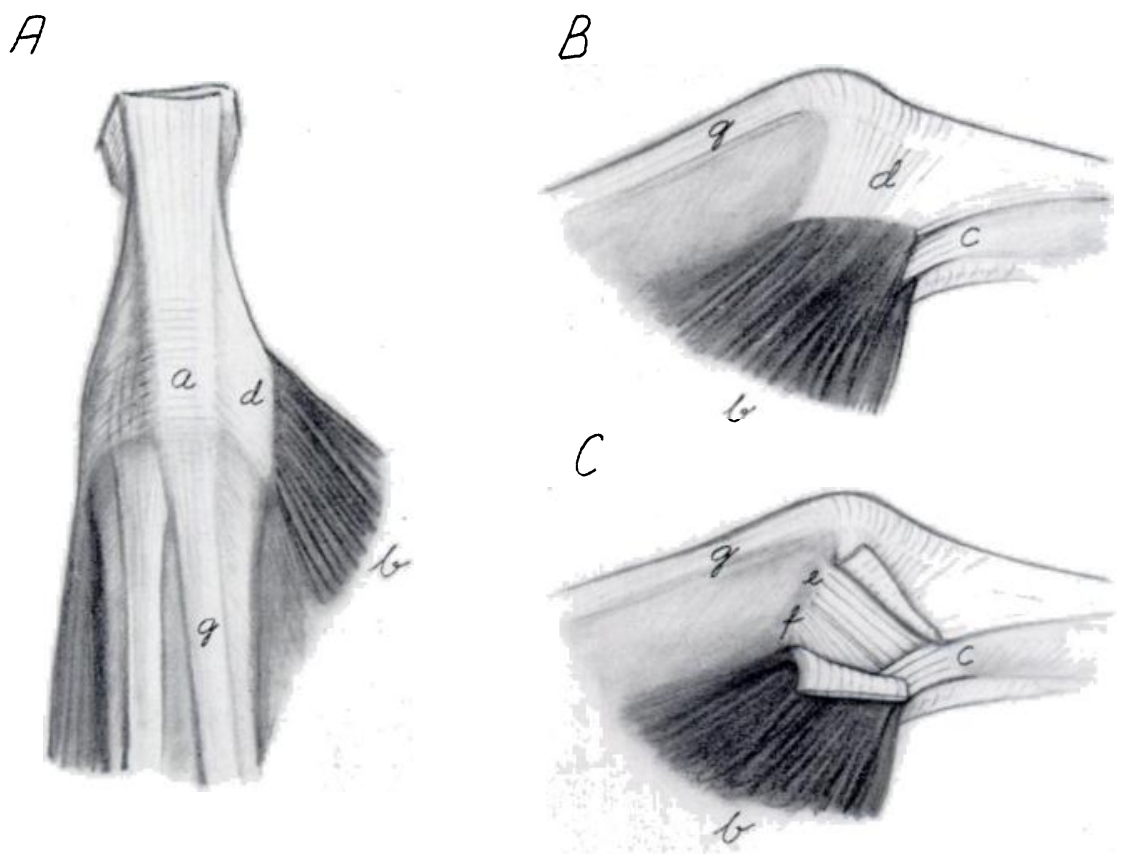

Fig. 1

The dorsal (A) and ulnar (B and C) aspects of the metacarpo-phalangeal joint of the right thumb. $\quad a=$ dorsal aponeurosis, $b=$ adductor pollicis, $c=$ tendon of adductor pollicis $d=$ adductor aponeurosis (see text), $e=$ collateral ligament proper, $f$ accessory collateral ligament, $g=$ tendon of extensor pollicis longus.

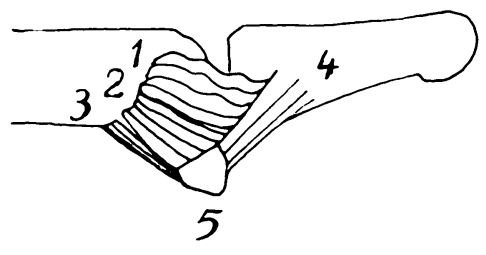

Fig. 2

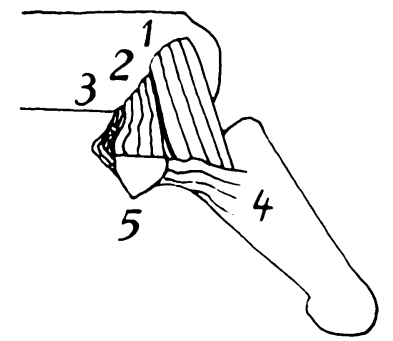

When the joint is fully extended the collateral ligament proper is relaxed whereas the accessory ligament is taut; at maximum flexion the reverse is true. 1 collateral ligament proper, $2=$ accessory collateral ligament, 3 and 4 -palmar ligaments, 5 - sesamoid bone.

Through the muscle that it serves, the adductor aponeurosis is of importance for the active stabilisation of the joint against violence tending to abduct the thumb, but it is of no value as a passive obstacle to abduction. If the adductor aponeurosis alone is severed there is no change in lateral stability; in contrast, severance of the ulnar collateral ligament makes abnormal abduction possible even if the adductor aponeurosis is left intact. 
When the joint is fully extended the collateral ligament proper is relaxed while the accessory ligament is taut (Fig. 2); in full flexion the reverse is true. If the ulnar collateral ligament proper is severed and the accessory ligament is left intact the stability of the extended joint will not be impaired, whereas increased abduction will be possible when the joint is flexed. This increase is, however, rather slight and does not become considerable until the accessory ligament has also been severed. Even then the abnormal mobility will be most apparent in flexion: in extension, abduction is restricted by the palmar ligaments so long as they are intact. If only the proper and accessory ulnar collateral ligaments are severed and an attempt is made to abduct the thumb as far as possible, the movement is bound to be combined with flexion and supination. It seems that the remaining intact ligaments and the shape of the skeletal parts involved cooperate to this end.

\section{A COMMON TYPE OF SEVERE DISPLACEMENT OF THE RUPTURED LIGAMENT}

Figure 3 is a semi-diagrammatic illustration of a case in which the ruptured ulnar collateral ligament is severely displaced. Having been torn from its distal attachment, the ligament has been folded over to point proximally. The upper picture shows how the ruptured end sticks out well proximal to the proximal edge of the adductor aponeurosis. The lower picture shows the adductor aponeurosis severed and folded back to reveal the site of the rupture on the phalanx. (If the rupture occurs more proximally-for instance, between the intermediate and distal thirds of the ligamentthe folded part of the ligament will be shorter but the picture will be the same in principle.) The degree to which the ligament is folded may vary: the ruptured end can point distally and ulnarwards, directly ulnarwards, or proximally and ulnarwards. A characteristic feature of every type, however, is that the ruptured end of the ligament has passed the proximal edge of the adductor aponeurosis so that the aponeurosis has become interposed between this end and the site of its attachment to the phalanx. Such interposition was observed in twenty-five of the thirty-nine cases.

Fig. 3

The ulnar aspect of the metacarpo-phalangeal joint of the right thumb. After distal rupture the ulnar collateral ligament has been folded right over. The torn end sticks out proximal to the adductor aponeurosis (shown divided in the lower picture).

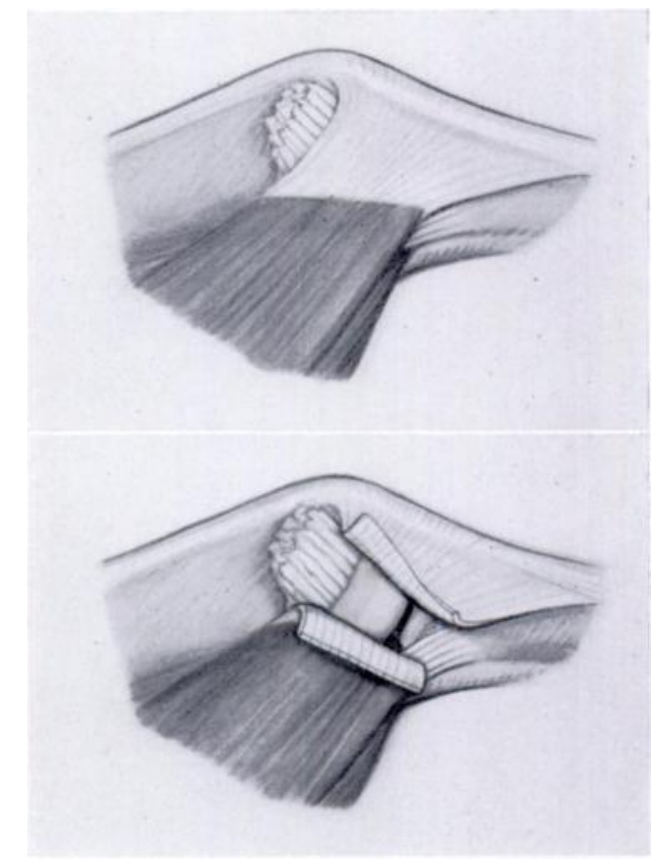

Fig. 3

\section{A TENTATIVE EXPLANATION OF THE DISPLACEMENT OF THE RUPTURED LIGAMENT}

Figure 4 illustrates a possible explanation of the way in which the ligament becomes displaced after distal rupture. The pictures show the metacarpo-phalangeal joint of the right thumb as seen from the dorsal side. In the initial position (A) the ulnar collateral ligament is hidden by the adductor aponeurosis, while the radial is exposed. At the instant of rupture (B) the adductor aponeurosis covers the ligament and prevents it from being folded over. Once the rupture has occurred, however, the torn end of the ligament can pass the proximal edge of the adductor aponeurosis (C), and when the phalanx then returns the ligament may be

VOL. 44 B, NO. 4, NOVEMBER 1962 
caught by the aponeurosis and folded over to point more proximally (D). The situation illustrated in Figure $4 \mathrm{C}$ can easily be reproduced in a post-mortem specimen by combined abduction, flexion and supination at the joint, after the proper and accessory ulnar collateral

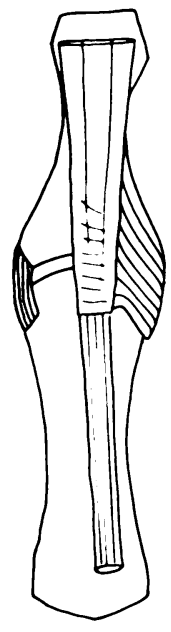

A

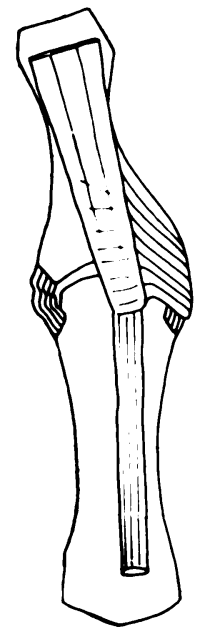

B

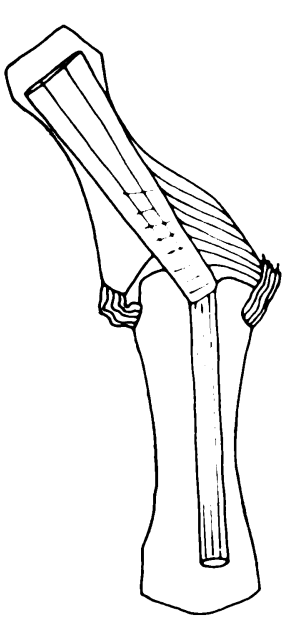

C

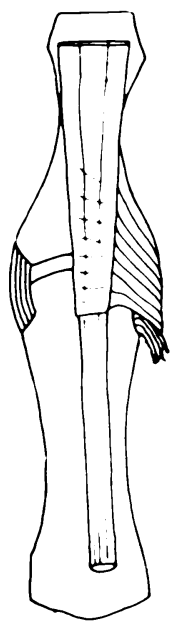

$D$

Fig. 4

The dorsal aspect of the metacarpo-phalangeal joint of the right thumb, showing skeletal parts, the collateral ligaments, the tendon of extensor pollicis longus and the adductor aponeurosis (see text).

ligaments have been severed at their distal attachments without injuring the adductor aponeurosis. To produce the effect illustrated in Figure $4 \mathrm{D}$ one must, while returning the phalanx, apply tension to the adductor aponeurosis-as for instance by pulling on its muscle, the adductor pollicis.

\section{ILLUSTRATIVE CASES}

A number of cases will be described in order to illustrate variations in the pathological anatomy of this injury.

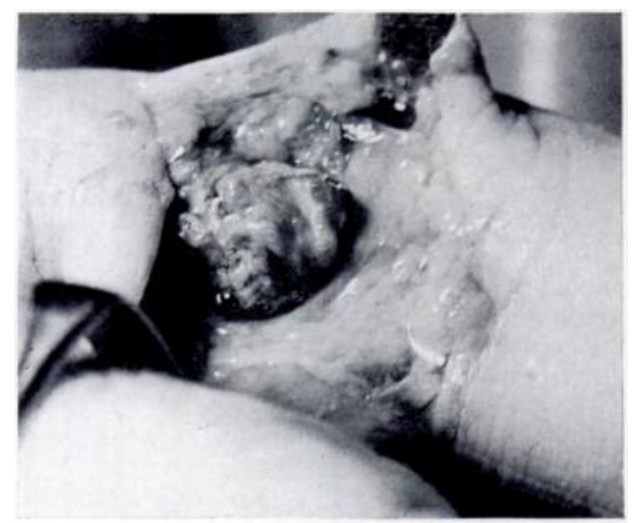

Fig. 5

Case 1-The metacarpo-phalangeal joint of the right thumb seen from the ulnar side. The ulnar collateral ligament, torn from its distal attachment, is displaced in accordance with the principle demonstrated in Figure 3 . The ruptured end sticks out proximal to the adductor aponeurosis.
Case 1-A forty-three-year-old man was examined a week after he had suffered violent abduction of the right thumb. A tender swelling was noted on the ulnar side of the metacarpal head, and the metacarpo-phalangeal joint was unstable upon abduction. At operation the following day it was found that the ulnar collateral ligament, torn from its distal attachment, had been folded over to point proximally (Fig. 5). The adductor aponeurosis, which was found intact, was severed at right angles to the line of its fibres. The accessory collateral ligament had ruptured near its proximal attachment. The collateral ligament proper was fastened to the base of the phalanx, using the "pull-out-wire " technique (Moberg and Stener 1953), the accessory ligament was realigned and the adductor aponeurosis was reconstructed with fine silk sutures.

Case 2-A sixty-four-year-old man sustained violent abduction of the right thumb in an accident with his autocycle. When examined fifteen days later he had a large swelling on the ulnar side of the 
metacarpal head, and the stability of the metacarpo-phalangeal joint upon abduction was impaired. Operation was undertaken the next day. Figure 6 shows the operation field after removal of granulation tissue. The ulnar collateral ligament had ruptured at its distal attachment and been folded right over to point proximally; at this stage, after sixteen days, it had already begun to adhere to the underlying tissue but could easily be dissected free and unfolded. After the adductor aponeurosis had been severed the ligament was fastened to the phalanx by a pull-out wire.

Comment-Cases 1 and 2 conform with the schematic explanation illustrated in Figure 3 and 4 D. In a further six cases with distal rupture the ligament was found to be in the same position, completely folded over and pointing proximally.

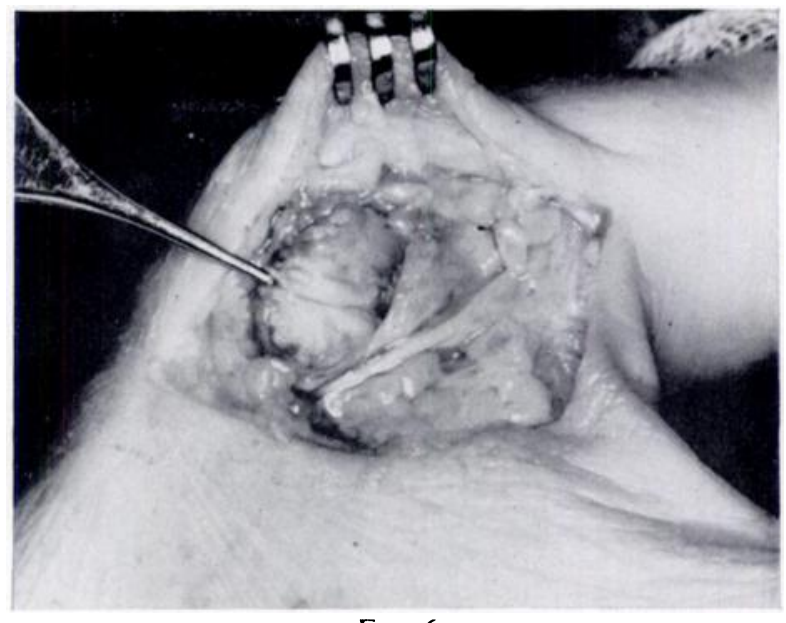

FIG. 6

Case 2-After distal rupture the ulnar collateral ligament has been folded over to point proximally. With the forceps a light pull is being exerted on the ligament in order to demonstrate the proximal edge of the adductor aponeurosis (in the centre of the operation field). Note the thumb's ulnar cutaneous branch from the radial nerve palmar to the ligament and crossing the aponeurosis.

Case 3-A seventeen-year-old man fell during gymnastics and injured his right thumb. On examination two days later there was tenderness on the ulnar side of the metacarpal head, and the proximal phalanx could be abducted at least 45 degrees. Radiographs showed a bone fragment well to the ulnar side of the metacarpal head; this fragment clearly derived from the base of the phalanx (Fig. 7). At operation four days after the accident it was found that the bone fragment was attached to the distal end of the ulnar collateral ligament. The fragment was fastened in its proper place by a pull-out wire.

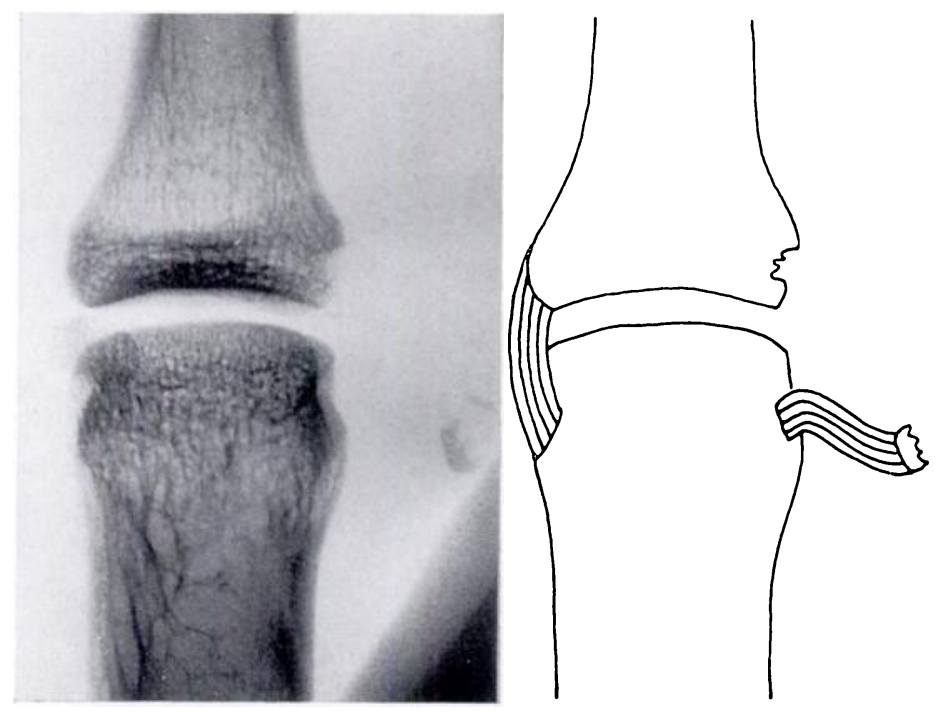

Fig. 7

Case 3-The ulnar collateral ligament has torn a fragment of bone from the base of the phalanx.

Comment-In this case displacement of the ligament was suggested by radiographic examination. A similar indication of the ligament's abnormal position was shown by radiography in a further four recent cases and in three older cases (Case 7). In four other

VOL. 44 B, NO. 4, NOVEMBER 1962 


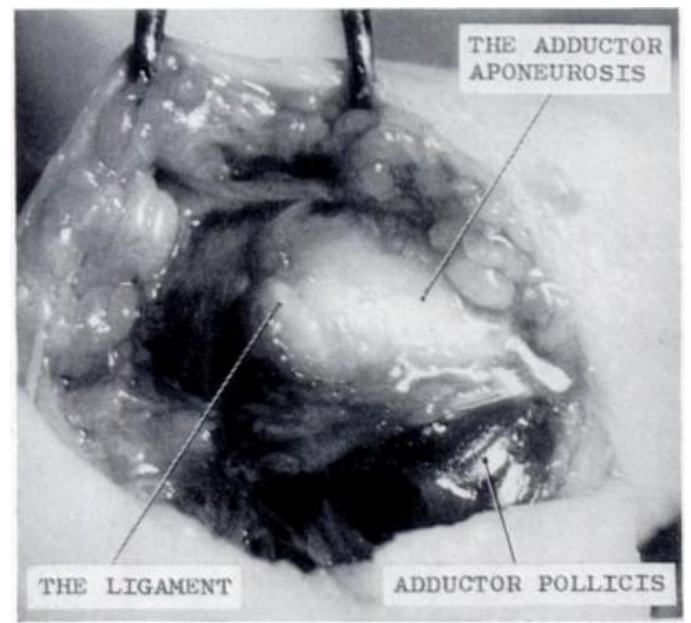

FIG. 8

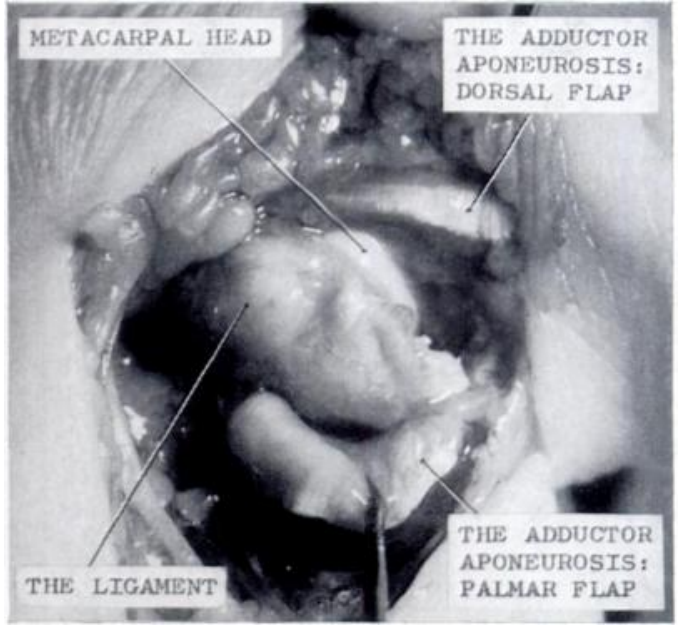

FIG. 9

Case 4. Figure 8-The metacarpo-phalangeal joint of the right thumb seen from the ulnar side. After distal rupture the ulnar collateral ligament causes a bulge in the fascia close to the proximal edge of the adductor aponeurosis. Figure 9-The metacarpo-phalangeal joint of the right thumb seen from the ulnar side. The adductor aponeurosis has been severed and its palmar flap folded back. Distal rupture of the ulnar collateral ligament, with a considerable gap between the ruptured end and the site of its attachment on the phalanx.

cases in which the ligament had torn out a fragment of bone this lay close to the phalanx but was rotated from 45 to 90 degrees.

Case 4-A twenty-three-year-old woman fell off a horse and injured her right thumb. On examination five days later the metacarpo-phalangeal joint proved to be unstable upon abduction. At operation the following day it was found that the ulnar collateral ligament bulged under the fascia just next to the proximal edge of the adductor aponeurosis (Fig. 8). Once the aponeurosis, which was intact, had been severed (Fig. 9) the ligament was found ruptured at its distal attachment and retracted in a proximal direction (its dorsal part most-five or six millimetres) but not folded over; the ruptured
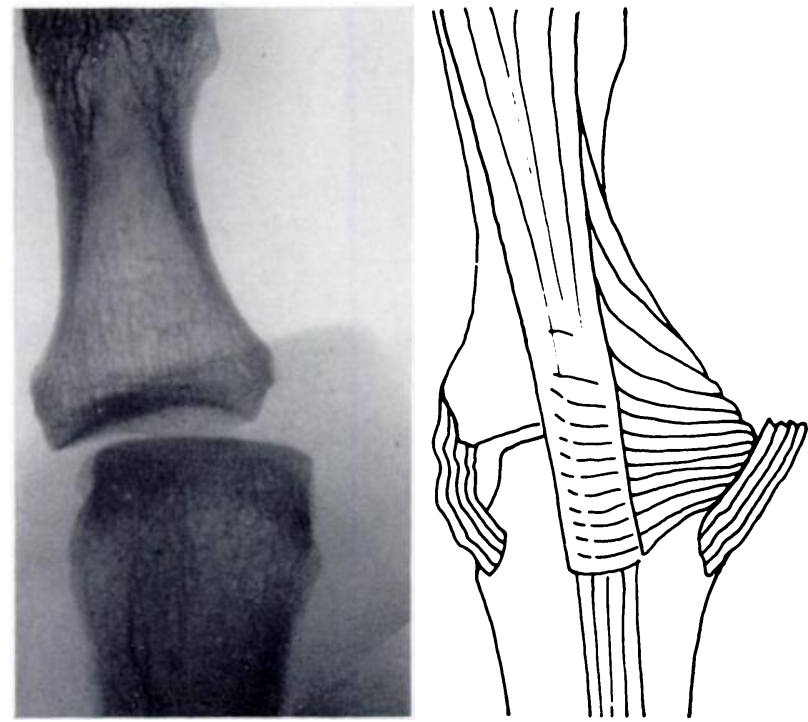

Fig. 10

Case 5-The ulnar collateral ligament has been torn from its distal attachment and been displaced outside the adductor aponeurosis. The radial subluxation of the joint could not be reduced until the adductor aponeurosis had been severed; the aponeurosis had got caught between the metacarpal head and the ligament. end pointed distally and was still under the adductor aponeurosis. The bulge under the fascia had been caused by the proximal part of the ligament. The ligament was realigned and fastened with two silk sutures to the tendon of adductor pollicis (Fig. I c) near the phalanx. The adductor aponeurosis was then reconstructed.

Comment-In a similar case the distally ruptured ligament had been retracted so far that the torn end was on a level with the proximal edge of the adductor aponeurosis, but was still under this.

Case 5-A fifty-year-old man fell and suffered violence to the right thumb. On examination the same day there was instability of the metacarpophalangeal joint upon abduction, and radiographs showed radial subluxation of the joint (Fig. 10). Complete reduction of the thumb was not possible before operation. At operation the day after the accident the 
ulnar collateral ligament was found to have ruptured distally and now lay outside the adductor aponeurosis. Reduction had not been possible because the adductor aponeurosis had got caught between the metacarpal head and the ligament. After the aponeurosis had been severed there was no obstacle to reduction.

Comment-The material included a further two recent cases in which radial subluxation of the joint was noted radiographically and in which at operation the adductor aponeurosis was found to have been caught up in this way.

Case 6-A sixty-four-year-old woman injured her left thumb when she fell forwards. Examination disclosed irreducible radial displacement of the proximal phalanx in relation to the metacarpal bone, and the patient was unable actively to extend either the interphalangeal or the metacarpo-

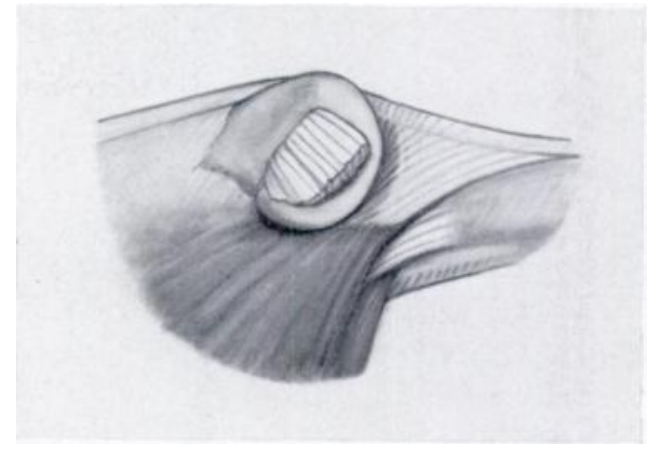

Fig. 11

Case 6-Distal rupture of the ulnar collateral ligament. Radial dislocation of the proximal phalanx. The greater part of the adductor aponeurosis lies between the two surfaces of the joint. The extensor tendons have slid down on to the radial side of the metacarpal head. phalangeal joint. The finding at operation four days after the accident is illustrated in Figure 11. The metacarpal head lay exposed just beneath the skin together with the ulnar collateral ligament, which had been torn off at its distal attachment. The adductor aponeurosis had become interposed between the two surfaces of the joint. The extensor tendons had slid down on to the radial side of the metacarpal head.

Comment-What happened in this injury was probably that the adductor aponeurosis, as a result of the severe dislocation of the proximal phalanx (Fig. $4 \mathrm{C}$ ), slid over the head of the joint and was caught by this when the phalanx returned.

When the ligament ruptures near its middle one sometimes finds that the distal fragment has got caught between the two surfaces of the joint (Stener 1959). In such a case the mechanism may well be the same as when the adductor aponeurosis gets caught in the joint.

Case 7-A thirty-three-year-old woman suffered a severe abduction injury to her right thumb in about 1950 . Treatment was by splintage. The pain gradually disappeared and she was able to do her housework as usual. In a mild injury in December 1960 the thumb was dislocated and pointed
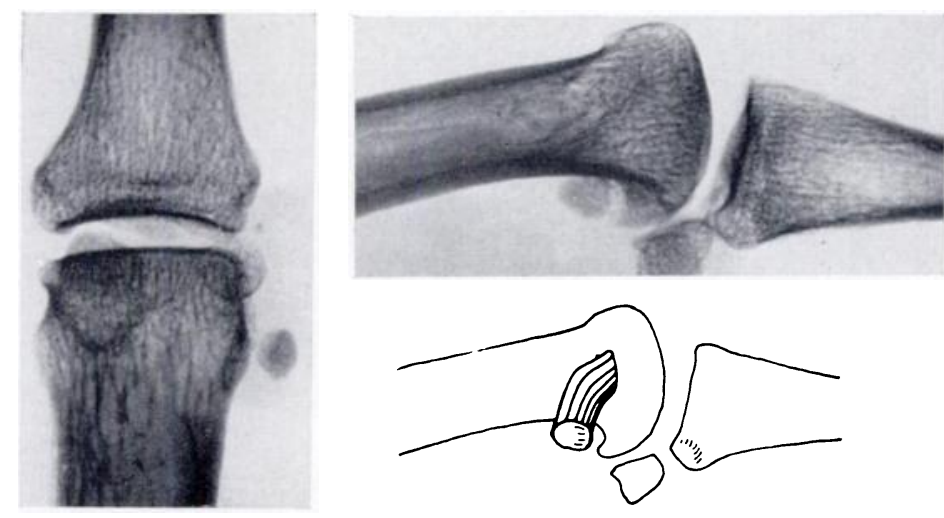

Fici. 12

Case 7-The ulnar collateral ligament has torn a fragment of bone from the base of the phalanx; the fragment is severely displaced in a proximal direction (injury ten years old).

almost straight out radially. The dislocation was reduced by a doctor. Fourteen days later the patient was admitted for operation. On examination it was found that the proximal phalanx could be abducted almost 80 degrees and there was no strength in the grip between the thumb and the index finger. Radiographs revealed a rounded bone fragment ulnar and palmar to the metacarpal head; and there

VOL. 44 B, NO. 4, NOVEMBER 1962 


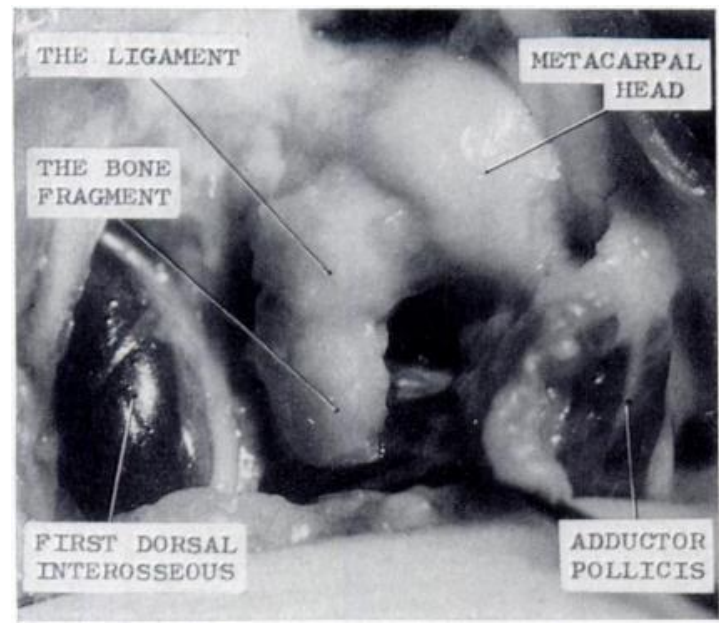

Fig. 13

Case 7-The metacarpo-phalangeal joint of the right thumb seen from the ulnar side. The adductor aponeurosis has been severed, and the scar tissue that surrounded the ligament and the bone fragment has been cut away. was a corresponding defect at the ulnar edge of the base of the phalanx (Fig. 12). This fracture was clearly a result of the earlier injury and was taken as an indication that the ulnar collateral ligament had torn off a fragment of bone at its distal attachment. At operation this interpretation of the radiographs proved to be correct. Figure 13 shows the ligament as it was found folded proximally and palmarwards and with the bone fragment attached. After the ligament had been unfolded the bone fragment was fastened to the phalanx with a pull-out wire (a hole was made in the fragment). Nine months later the joint was stable and the grip between the thumb and the index finger was equal to that on the uninjured side.

Comment-It is remarkable that the ligament could still be found ten years after the injury. In a further three cases a severely displaced ligament could be isolated even though the injury was not recent (five months, nine months, and eight years old).

Case 8-A seventy-five-year-old man fell off his autocycle and injured his right thumb. Rupture of the ulnar collateral ligament of the metacarpo-phalangeal joint was diagnosed and he was operated upon the day after the accident. The joint could be abducted 60 degrees (without anaesthetic). It was found that the collateral ligament proper had ruptured at its proximal attachment whereas the accessory ligament had been torn off distally. Only the latter ligament was displaced; the picture was typical, with the ruptured end pointing proximally and sticking out beyond the proximal edge of the adductor aponeurosis.

Comment-With this one exception, either both ligaments had ruptured distally or the collateral ligament proper had ruptured distally and the accessory ligament more proximally. In only two other cases was the collateral ligament proper ruptured at its proximal attachment - that is, in three out of the thirty-nine consecutive cases (in thirty-three of which the rupture was distal).

Case 9-A thirty-eight-year-old man had his left thumb forcibly abducted and flexed. He displayed haematoma and tenderness on the ulnar side of the metacarpo-phalangeal joint; the proximal phalanx could be deviated abnormally in a radio-palmar direction. At operation three days after the accident the proximal half of the adductor aponeurosis was found ruptured transversely in relation to the line of its fibres (Fig. 14); moreover, the ulnar collateral ligament was totally ruptured at its distal attachment, but not markedly retracted.

Comment-This was the only case in which the adductor aponeurosis was found ruptured. It cannot, however, be ruled out that such an injury had occurred in the older cases in addition to the rupture of the ligament.

\section{DISCUSSION}

Restored function after operative treatment of recent total rupture of the ulnar collateral ligament of the metacarpo-phalangeal joint of the thumb has been reported by Moberg and

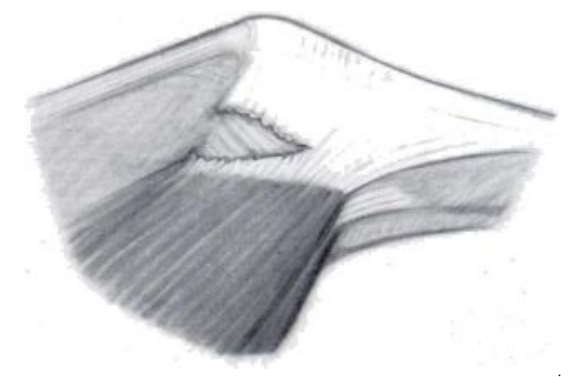

FIG. 14

Case 9-Partial rupture of the adductor aponeurosis. There was also total rupture of the ulnar collateral ligament at its distal attachment. 
Stener (1953), Frykman and Johansson (1956) and Strandell (1959). The same results were obtained in the present series, but it has been my intention rather to communicate observations made concerning the pathological anatomy of rupture of the ligament. These observations provide a strong argument in favour of operative treatment of this injury. Thus it can be concluded from the clinical material that the ligament, when ruptured distally-as is most frequently the case-is often displaced in such a way that the adductor aponeurosis becomes interposed between the ligament's ruptured end and the site of its attachment on the phalanx; the ligament is folded over to a greater or lesser extent and the ruptured end sticks out past the proximal edge of the adductor aponeurosis. Such displacement of the ligament with interposition of the adductor aponeurosis was found in twenty-five of the thirty-nine consecutive cases. In two cases in which the distally ruptured ligament was not folded over there was nevertheless a considerable gap between the ruptured end and the site of its attachment on the phalanx (Case 4).

Displacement of the ligament cannot be ruled out before operation. On the other hand there may be good reason to suspect that the ligament is displaced, especially when radiographs show a bone fragment torn from the base of the phalanx, with the fragment displaced proximally and ulnarwards (Cases 3 and 7). Irreducible subluxation of the joint suggests a pathological anatomy similar to that in Case 5 or Case 6.

Another feature that suggests displacement of the ligament is the presence of a tender swelling on the ulnar side of the metacarpal head. This swelling may be caused by the displaced ligament itself and the wealth of granulation tissue that forms around the ligament within a few days of the accident. In old and untreated cases this tissue may give rise to a voluminous callus on the ulnar side of the joint. In so far as the proximal phalanx is displaced radially, the metacarpal head also contributes to the size of the swelling.

That the most pronounced tenderness on palpation is located to the level of the metacarpal head is by no means a certain indication that the rupture is proximal; it is as likely to be distal with the ligament folded over to point proximally.

Normal lateral stability in extension does not preclude rupture of the ulnar collateral ligament; such a rupture as an isolated injury is best demonstrated by comparing combined abduction, flexion and supination on the injured and uninjured sides. Pronounced instability is an indication that both the accessory ligament and the collateral ligament proper have ruptured.

Familiarity with the various ways in which the ruptured ligament may be displaced facilitates the exposure of the lesion. This is particularly the case when the injury is not recent, because the formation of granulation tissue soon obscures the pathological anatomy. I have found it advisable to start the dissection by isolating the adductor aponeurosis. This will usually make it clear whether the torn end of the ligament has been displaced outside the aponeurosis or not. The next step-assuming the rupture is not proximal-is to sever the adductor aponeurosis at right angles to the line of its fibres (Fig. 9). This will disclose the location of the rupture, and the ligament, if it has been displaced, can be returned to its proper position. The palmar flap of the aponeurosis should be retracted so that one can determine whether or not the accessory collateral ligament has ruptured as well. If the lesion is not fully exposed there is a risk that, when the collateral ligament proper has ruptured distally and the accessory proximally, one may sew the former to the latter with unsatisfactory stability as a result. It may also happen that if the ligament is folded right over the rupture is judged to be proximal instead of distal, in which case the ruptured end may be sewn in error to the periosteum of the metacarpal bone.

Naturally, direct suture is employed if the rupture is situated some way from the bone. In certain cases this method can also be used when the ligament has parted just where it attaches to the phalanx; the ligament is then fastened with one or two silk sutures to the tendon of adductor pollicis (Fig. $1 \mathrm{c}$ ), the role of the sutures here being to hold the ligament close to

V OL. 44 B, NO. 4, NOVEMBER 1962 
the phalanx while the injury heals. This method was used, for example, in Case 4, with restored function as a result.

At operation it is important not to injure the thumb's ulnar cutaneous branch of the radial nerve (Fig. 6); this branch may be concealed by the granulation tissue which forms around the displaced ligament.

After suture of the ligament the adductor aponeurosis should be carefully reconstructed, whether it has been severed intentionally during the operation or torn wholly or partly at the time of the injury. Case 9 was the only one in the material in which the adductor aponeurosis was found ruptured. No such rupture, observed and operated upon soon after its occurrence, has been found in the literature; older cases have, however, been described (Mondry 1940, Kaplan 1960, 1961). In the present case the ulnar collateral ligament was found ruptured as well; this ligament was said not to have been ruptured in the four cases reported by Mondry. Because Mondry made his observations at a relatively late stage the possibility remains, however, that the ligament, having been ruptured, had healed meanwhile with elongation. In Kaplan's two cases an unsuccessful attempt had previously been made to restore the function of the joint on the assumption that the instability was due to rupture of the ulnar collateral ligament. Kaplan's opinion that radial subluxation at the metacarpo-phalangeal joint of the thumb depends not upon injury to the ulnar collateral ligament but upon rupture of the "dorsal apparatus" (according to his drawings the same as rupture of the adductor aponeurosis) is not confirmed by the present clinical material, nor by the observation on the post-mortem material that the lateral stability of the joint was unimpaired after only the adductor aponeurosis had been severed.*

In order to ensure good results with the operation it is important to use a suitable plaster technique. A plaster enclosing the thumb, the hand as far as the proximal transverse skin crease and the forearm should be applied. Special care should be taken to see that the plaster is moulded in such a way as to eliminate the possibility of ulnar deviation of the first metacarpal by the first dorsal interosseous muscle; otherwise this muscle may achieve indirect abduction at the metacarpo-phalangeal joint since the thumb-enclosed in its plaster-is prevented from moving with the metacarpal bone. Plaster treatment by itself, however adequate the technique, will not give satisfactory results in the majority of cases (Wosnik 1958).

\section{SUMMARY}

1. The pathological anatomy of total rupture of the ulnar collateral ligament of the metacarpophalangeal joint of the thumb has been studied at operation in thirty-nine patients.

2. It has been found that the ligament, when ruptured distally (the most usual type) is often displaced so that the ulnar expansion of the dorsal aponeurosis-here referred to as the adductor aponeurosis-becomes interposed between the ruptured end of the ligament and the site of its attachment on the phalanx; the ligament gets folded over to a greater or lesser extent and the ruptured end sticks out beyond the proximal edge of the adductor aponeurosis. Such displacement of the ligament with interposition of the adductor aponeurosis was found in twenty-five of the thirty-nine cases. The ligament may also be displaced without the adductor aponeurosis being interposed (with a gap between the ruptured end and the site of its attachment on the phalanx).

3. Variations in the pathological anatomy are illustrated by more detailed descriptions of nine cases. In one of these the adductor aponeurosis had got caught between the two surfaces of the joint, and in another the adductor aponeurosis was found ruptured as well as the ulnar collateral ligament.

\footnotetext{
* It is theoretically possible, however, that an isolated rupture of the adductor aponeurosis might lead, as a result of insufficient active protection against abduction, to gradual elongation of the ulnar collateral ligament and hence to radial subluxation at the joint.
} 
4. On the basis of dissection studies on forty-two fresh post-mortem specimens, a rational explanation is given of the pathological anatomy.

5. The results of the present investigation on clinical and anatomical material provide a strong argument in favour of operative treatment for total rupture of the ulnar collateral ligament of the metacarpo-phalangeal joint of the thumb.

6. Aspects of diagnosis and operative technique are discussed.

This study was supported by grants from Göteborgs Läkarsällskap. I wish to thank Dr Svante Edshage for taking some of the photographs.

\section{REFERENCES}

Frykman, G., and Johansson, O. (1956): Surgical Repair of Rupture of the Ulnar Collateral Ligament of the Metacarpo-Phalangeal Joint of the Thumb. Acta Chirurgica Scandinavica, 112, 58.

KAPLAN, E. B. (1960): Lateral Subluxation of the Metacarpophalangeal Joint of the Thumb: Experimental Study. Bulletin of the Hospital for Joint Diseases, 21, 200.

Kaplan, E. B. (1961): The Pathology and Treatment of Radial Subluxation of the Thumb with Ulnar Displacement of the Head of the First Metacarpal. Journal of Bone and Joint Surgery, 43-A, 541.

MoberG, E., and Stener, B. (1953): Injuries to the Ligaments of the Thumb and Fingers. Diagnosis, Treatment and Prognosis. Acta Chirurgica Scandinavica, 106, 166.

MoNDRY, F. (1940): Beitrag zur operativen Behandlung des Wackeldaumens. Zentralblatt für Chirurgie, 67, 1,532 .

Stener, B. (1959): Discussion. Nordisk Medicin, 62, 1,165.

STENER, B. (1962): Hyperextension Injuries to the Metacarpo-phalangeal Joint of the Thumb-Rupture of Ligaments, Fracture of Sesamoid Bones, Rupture of Flexor Pollicis Brevis. An Anatomical and Clinical Study. (To be published in Acta Chirurgica Scandinavica.)

Strandell, G. (1959): Total Rupture of the Ulnar Collateral Ligament of the Metacarpophalangeal Joint of the Thumb. Results of Surgery in 35 Cases. Acta Chirurgica Scandinavica, 118, 72.

Wosnik, H. (1958): Die geschlossenen Bandverletzungen des Daumengrundgelenks. Hefte zur Unfallheilkunde $56,45$. 